\title{
Influence of Sputter Deposition Time on the Growth of $c$-Axis Oriented AIN/Si Thin Films for Microelectronic Application
}

\author{
V. VasanthiPillay ${ }^{1}$, K. Vijayalakshmi ${ }^{2 *}$ \\ ${ }^{1}$ Department of Physics, Sujatha Degree and PG College for Women, Hyderabad, India \\ ${ }^{2}$ Department of Physics, Bishop Heber College, Tiruchirappalli, India \\ Email: v.vasanthipillayphy@gmail.com, *viji71naveen@yahoo.com
}

Received April 27, 2012; revised June 7, 2012; accepted June 30, 2012

\begin{abstract}
Aluminum Nitride films were grown on and Si (100) substrate by DC reactive magnetron sputtering at room temperature. Influence of sputter deposition time on properties of the AlN films was studied. Structural optical and electrical properties of the film were investigated. XRD measurements showed the presence of hexagonal wurtzite structure. The optical reflectance spectra of the film were taken and the band gap calculated varied from 4.35 to $5.3 \mathrm{eV}$. Finally MIS capacitors were fabricated on silicon substrates and variation of dielectric parameter with deposition time was reported.
\end{abstract}

Keywords: Aluminum Nitride; Thin Films; Sputtering; Preferential Orientations

\section{Introduction}

Aluminum Nitride (AIN) and its alloys are the most attractive material investigated over the past several years [1-3]. Growth of high-quality epitaxial wurtzite ALN thin film on different substrates have been of great interest due to their peculiar features such as high electrical resistivity $\left(10^{11}-10^{13} \mathrm{ohm} \cdot \mathrm{cm}\right)$, high thermal conductivity, high hardness (11 - $15 \mathrm{GPa}$ ), wideband gap 6.0 - 6.2 $\mathrm{eV}$ and high velocity of acoustic waves etc. The combination of these characteristics makes AlN thin films a promising material for many electronic, optoelectronic, acoustic devices such as surface acoustic wave (SAW) $[4,5]$. The characteristics of AIN films are greatly influenced by their microstructure. Growth of smooth surface and defect less structure were the goal of device development. Various deposition techniques and substrates have been employed in an attempt of achieving high quality growth; the most frequently used substrates are $\mathrm{Al}_{2} \mathrm{O}_{3}, \mathrm{SiC}, \mathrm{Si}$ (111) and $\mathrm{Si}(100)$. The later is of special interest because of the possibility to integrate with the contemporary Si device technology for different functions, e.g. UV detection or emission and Si-ICs on a common substrate.

In the past years several methods have been developed to prepare AlN thin films, such as chemical vapor deposition (CVD), molecular beam epitaxy (MBE), pulsed laser deposition (PLD), ion-beam assisted deposition and

"Corresponding author. reactive sputtering [6-9]. However, these methods are expensive and the required high temperatures to reach satisfying properties are often incompatible with microelectronic processes. Among other techniques, the reactive magnetron sputtering process (RMS) is an attractive deposition technique because it presents advantages of being low temperature and low cost methods, and it allows fine-tuning of the material characteristics [10-12].

\section{Experimental}

AlN films were deposited with a $99.5 \%$ pure $\mathrm{Al}$ target DC magnetron sputtering system operated at $60 \mathrm{~W}$ DC cathode power and in pure Ar and $\mathrm{N}_{2}$ gas mixture, which was introduced into the chamber by separate mass flow controllers. The deposition of ALN films can take place in a wide range of temperatures from room temperature up to $400^{\circ} \mathrm{C}$. High temperature deposition has the disadvantage of producing degradation of the substrate, incorporation of impurities and thermal damage to the growing film. Hence deposition of AlN films at low temperature has become increasingly important and value [13]. Therefore AlN films were grown on (100) oriented silicon substrate at room temperature. Degreasing of glass was carried out ultrasonically in successive baths of acetone, ethanol and deionized water, and the native oxide on the silicon wafer was removed through an etching step in dilute HF solution. After this initial cleaning process, high purity Ar gas was introduced into 
the chamber and the chamber was evacuated to below $1 \times$ $10^{-5}$ mbar. Then prior to each run the target was presputtered with argon gas for 5 minutes with the target shutter closed. During pre sputtering DC power and Ar pressure was kept constant at $60 \mathrm{~W}$ and $1 \times 10^{-5} \mathrm{mbar}$ respectively, then nitrogen was introduced into chamber and reactive sputtering was initiated. AlN films were grown on Si substrate for different deposition time keeping the other deposition parameters constant. A summary of deposition parameters and ranges used in AlN thin films is listed in Table 1. For electrical measurements, Metal-insulator-semiconductor (MIS) structures were formed by sputter deposition through a metal mask of $\mathrm{Al}$ dots on AlN film as top electrode and a continuous Al film on Si wafer backside as bottom electrode.

The thickness of the films measured using stylus profilometer for 2, 4, 6, 8 minutes of deposition time were $49,81,102,114 \mathrm{~nm}$ respectively. The type of crystalline structure, orientation and grain size was examined by $\mathrm{X}$-ray diffraction (XRD). Optical studies were carried out by UV-visible spectrometer and band gap was calculated from the obtained reflectance spectra. Finally dielectric parameters were measured using impedance analyzer in frequency range $1 \mathrm{KHz}$ to $1 \mathrm{MHz}$ at room temperature.

\section{Results and Discussion}

\subsection{XRD Analysis}

AlN films were deposited on Si (100) substrate for different deposition time, keeping the sputtering pressure,

Table 1. AlN deposition parameters.

\begin{tabular}{|c|c|}
\hline Parameters & Values \\
\hline Deposition process & DC magnetron sputtering \\
\hline Magnetron & Disc \\
\hline Area of cathode (target), inch & 2 \\
\hline Material of the target & $\mathrm{Al}$ \\
\hline Discharge voltage, $\mathrm{V}$ & 510 \\
\hline Discharge current, $\mathrm{mA}$ & 117 \\
\hline Substrate & Silicon (100) \\
\hline Substrate temperature in Kelvin & 300 \\
\hline Working gases & $\mathrm{Ar}+\mathrm{N}_{2}$ \\
\hline Initial pressure in chamber, mbar & $1.3 \times 10^{-4}$ \\
\hline Partial pressure of active $\left(\mathrm{N}_{2}\right)$, mbar & $5 \times 10^{-5}$ \\
\hline Total pressure in the chamber, mbar & $1 \times 10^{-3}$ \\
\hline $\begin{array}{l}\text { Distance between the cathode and } \\
\text { anode, } \mathrm{cm}\end{array}$ & 4 \\
\hline Deposition time, minutes & $2,4,6,8$ \\
\hline
\end{tabular}

target power, substrate target distance and nitrogen concentration constant. XRD analysis were carried out using RIGAKU X-ray diffractometer employing $\mathrm{Cu} \mathrm{K}_{\alpha}(1.5406$ $\AA)$ radiation; $\theta$ - $2 \theta$ scans were performed with step size of 0.05 at a scan speed of $3 \mathrm{sec}$ per step in the range of $10-80$. The diffraction planes in $\theta-2 \theta$ method are parallel to the sample surface. Accordingly, this method is very helpful in studying preferred orientation in thin film materials when compared to grazing angle incidence method [15]. The influence of deposition time on the crystalline orientation of AlN films has been investigated. All the deposited films were found to have hexagonal wurtzite structure. Figure 1 shows the XRD pattern of AlN films on Si substrate for different deposition time. The micro structural properties and the lattice disorders are studied from the analysis of XRD peaks, and are listed in Table 2. The mean crystallite size was found to be $\sim 60$ $\mathrm{nm}$. By substituting the measured crystallite size, the strain in the films can be determined using the following equation $\varepsilon=(\beta / \tan \theta)-(\mathrm{k} \lambda / \sin \theta)$. In addition, it is evident that, both strain and dislocation density increases with increase in deposition time, which reveals the increase of particle collision due to the augmentation of stress in the lattice.

The crystal orientation of AlN films was strongly dependent on deposition conditions. It was found that under low deposition time of $2 \mathrm{~min}$ the film presents features at $32.98^{\circ}$ and $36^{\circ}$ corresponding to (100). As the deposition time increased to $4 \mathrm{~min}$ and $6 \mathrm{~min}$, the intensity of AlN (100) plane is decreased, while the intensity of AIN (002)

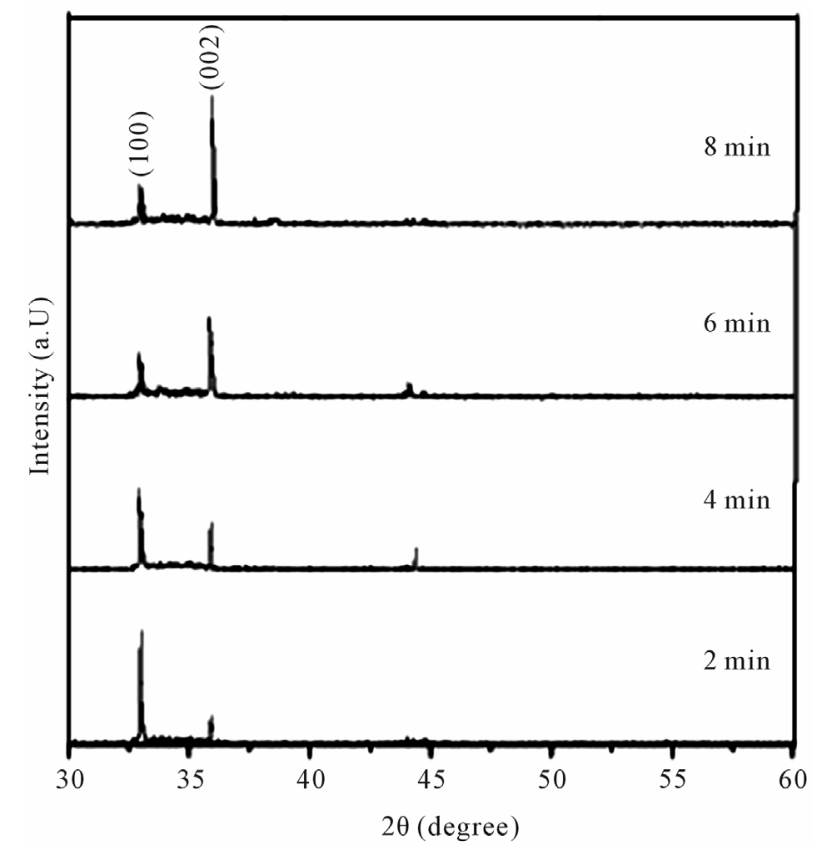

Figure 1. X-ray intensity vs. the diffraction angle $2 \theta$ of AIN thin films deposited on $\mathrm{Si}(100)$ substrate at different deposition time: (a) $2 \mathrm{~m}$; (b) $4 \mathrm{~m}$; (c) $6 \mathrm{~m}$; (d) $8 \mathrm{~m}$. 
Table 2. Structural parameters calculated from XRD data.

\begin{tabular}{ccccc}
\hline Time min & $\mathrm{d}$ spacing $\left(\mathrm{d}_{\mathrm{hkl}}\right) \AA$ & Crystallite size $(\mathrm{D}) \mathrm{nm}$ & Strain $(\varepsilon)$ & Dislocation density $(\delta)$ lines $/ \mathrm{m}^{2}$ \\
\hline 2 & 2.7137 & 61.40 & $4.48 \times 10^{-4}$ & 2.8492 \\
4 & 2.7105 & 61.40 & $4.82 \times 10^{-4}$ & 2.9491 \\
6 & 2.7121 & 57.47 & $5.47 \times 10^{-4}$ & 3.3807 \\
8 & 2.7121 & 57.47 & $5.78 \times 10^{-4}$ & 3.8147 \\
\hline
\end{tabular}

plane is enhanced. Further increasing the deposition time to $8 \mathrm{~min}$ resulted in films with highly oriented (002) (JCPDS file No. 00-0025-1133), with less intense AlN (100) plane.

The mechanism of preferential orientation of AlN film can be explained by the crystalline lattice structure of AlN. The hexagonal wurtzite structure of AlN has two kinds of AlN bonds named $B_{1}$ and $B_{2}$ [16]. The formation energy of the $B_{2}$ bond is larger than that of $B_{1} .\{100\}$ plane consists of only $B_{1}$ bonds, while $\{002\}$ and $\{101\}$ consist of $\mathrm{B}_{1}$ and $\mathrm{B}_{2}$ bonds together. Accordingly ad atoms with more energy favor the formation of $\{002\}$ surface plane. Another factor, which strongly influences the formation of preferred surface planes, is packing habit of such planes. For the development of close-packed surface planes the ad atoms need a longer time to accommodate themselves into the low energy configured lattice sites before the arrival of the next layer of reactive species. A low deposition rate is necessary in such cases [13]. Hence, we predict that at a deposition time of $8 \mathrm{~min}$, the coated film has low deposition rate, which favors the formation of highly oriented (002) preferential plane with enhanced crystal quality, which can provide good piezoelectric response.

\subsection{Optical Properties}

The optical reflectance of the films has been recorded employing a Perkin-Elmer Lamba-950 spectrometer. The optical energy band gap of AlN films is determined by analyzing the optical data with the expression for the reflectance and photon energy using the Tauc relation

$$
2 \alpha \mathrm{h} v=\mathrm{A}\left(\mathrm{h} v-\mathrm{E}_{\mathrm{g}}\right)^{\mathrm{n} / 2}
$$

where $\mathrm{n}$ is the constant, which is equal to one for direct band gap semi-conducting material and four for and indirect band gap semi-conducting material. $\mathrm{h} v$ is the photon energy, and $\alpha$ the absorption coefficient, which can be written in terms of reflectance as $2 \alpha t=\ln \left[\left(\mathrm{R}_{\max }-\right.\right.$ $\left.\mathrm{R}_{\min }\right) /\left(\mathrm{R}-\mathrm{R}_{\min }\right)$ ] where $t$ is the thickness of the sample and $\mathrm{R}$ the reflectance for any intermediate photon energy. Figure 2 shows the reflectance spectra of AlN thin film on $\mathrm{Si}$ substrate for different deposition time. A fall in reflectance is observed from $R_{\max }$ to $R_{\min }$ due to absorption of light by the material. Figure $\mathbf{3}$ shows, a graph is

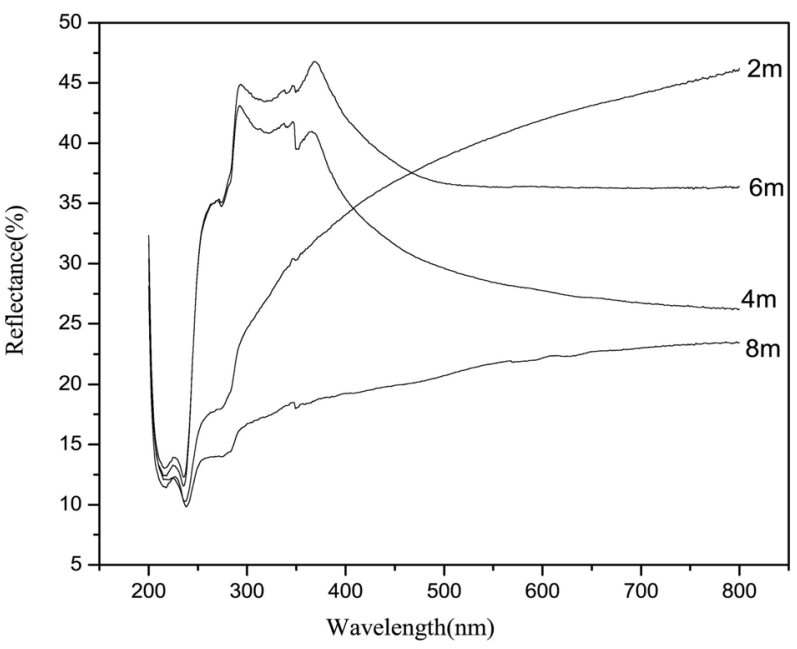

Figure 2. Reflectance spectra of AIN thin film on Si (100) at different deposition time: (a) $2 \mathrm{~m}$; (b) $4 \mathrm{~m}$; (c) $6 \mathrm{~m}$; (d) $8 \mathrm{~m}$ the films.

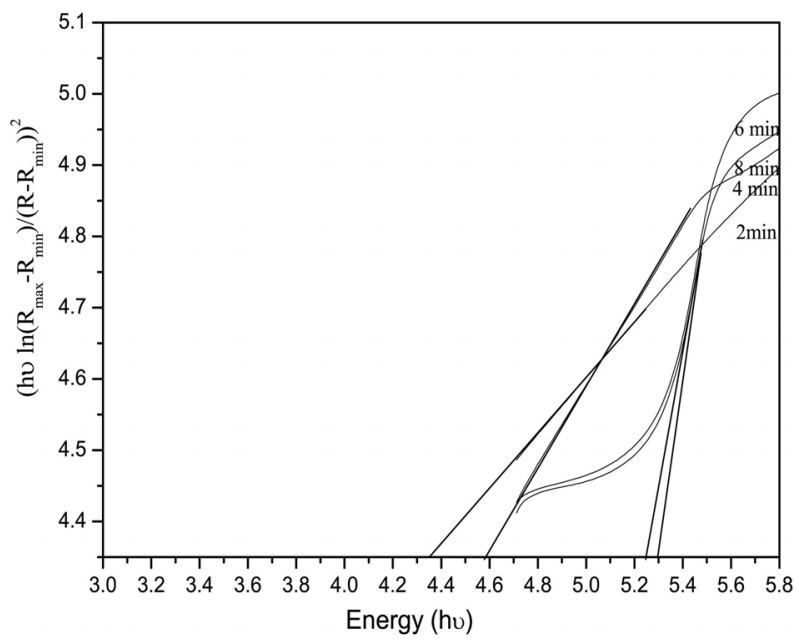

Figure 3. Graph between $(\alpha h v)^{2}$ and $h v$.

plotted between $(\alpha \mathrm{h} v)^{2}$ and $\mathrm{h} v$ using Equation (1). The extrapolation of linear portion of the plot of the energy axis $(\alpha h v)^{2}=0$ gives the value of the energy band gap of the thin film. It is found that the energy band gap of the AlN film varies from 4.35 to $5.3 \mathrm{eV}$. It is clear that the energy band gap of AlN films increases with increase in the deposition time. This variation in band gap is attributed to the internal strain in the films which increases 
with the deposition time as shown in the Table 2. The refractive index $(\eta)$ of the sputter-deposited AlN is dependent on the sputtering conditions since it is related to the composition and density of the films.

For polycrystalline AIN films obtained by DC reactive sputtering, the refractive index varies from 1.9 to 2.1 . The refractive index of our AIN samples, determined from the dielectric measurement of $\varepsilon$ and $\delta$, revealed that they are in the range of $1.1-1.2$, for the AlN samples prepared at a deposition time of 8 minutes. The decrease in the refractive index values is observed in the presence of impurities (such as oxygen) or nitrogen vacancies present in the film [17].

\subsection{Electrical Properties}

The variation of the dielectric parameters, such as $\mathrm{C}, \varepsilon$ and $\tan \delta$ of MIS capacitors with deposition time was studied in the frequency range from $10 \mathrm{KHz}-1 \mathrm{MHz}$ using impedance analyzer at room temperature. Plots of the dielectric constant $\varepsilon$, loss factor $\tan \delta$ and capacitance $\mathrm{C}$ as a functions of the frequency for different deposition time of AlN films are shown in Figures 4(a)-(c). The capacitance of the thin insulating film almost remains constant in the frequency range from $10 \mathrm{KHz}-1 \mathrm{MHz}$, with its value varying from $0.8 \times 10^{-8}$ to $1.8 \times 10^{-7} \mu \mathrm{F}$. It is observed that, as the deposition time increases the capacitance decreases with least value at 8 minutes of deposition time.

The dielectric constant of AlN films was between 6.0 and 6.8, which is similar with reported values for AIN [15]. The real part of dielectric constant increases with increasing deposition time and decreases with increasing frequency. The observed dependence of the dielectric constant on the dielectric film thickness (deposition time) for thinner films (less than $\sim 1000$ ) is attributed to defects such as voids, stresses, in homogeneity, grain boundaries, discontinuities etc, which are normally present in vacuum-deposited films, rather than to the non-stoichiometry of the deposits resulting from an excess of oxygen or metal atoms. Some of these defects are removed by selfannealing or aging processes, but others require more thermal energy such as is provided by an annealing process. As the films become thicker, the density of voids decreases, resulting in a higher value of dielectric constant, which evidently becomes thickness independent. Adam et al. [17] reported the dependence of dielectric constant on film thickness. The dielectric constant was between 4 and 11 for thicker layers $(\geq 100 \AA)$ and decreased to values between 2 and 6 for thicknesses below $100 \AA$ A. And Dimitrova et al. [18] reported low dielectric constants $(6.8-7.1)$ due to the presence of nitrogen vacancies in the AIN lattice.

The dielectric dissipation factor $\tan \delta$ of an insulating

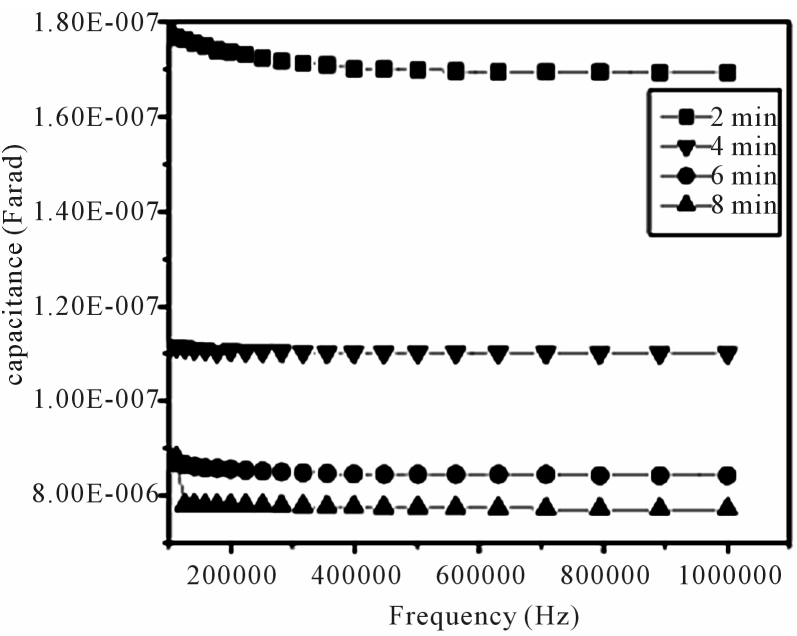

(a)

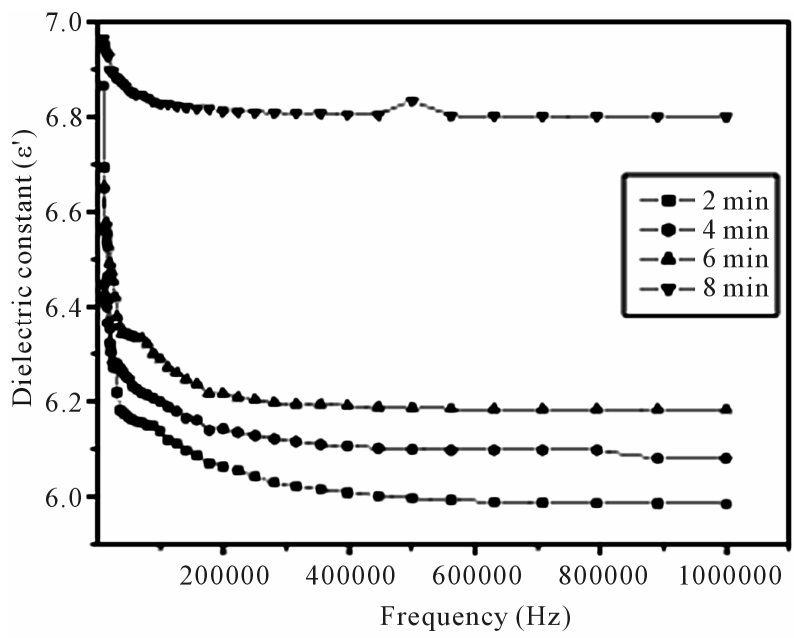

(b)

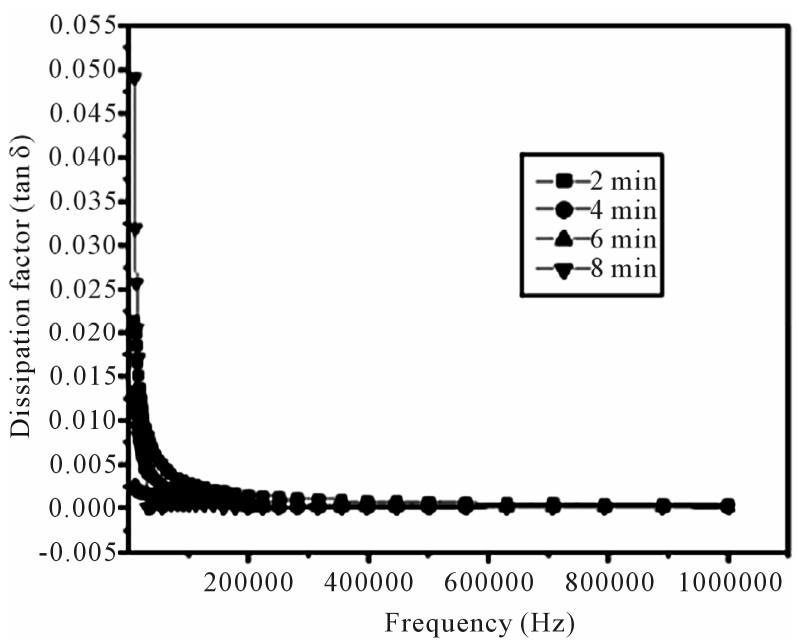

(c)

Figure 4. (a) Graph between capacitance and frequency for different deposition time; (b) Graph between dielectric constant and frequency for different deposition time; (c) Graph between dissipation factor and frequency for different deposition time. 
material is the tangent of the loss angle $\delta$. In a perfect dielectric, the voltage wave and the current are exactly $90^{\circ}$ out of phase. As the dielectric becomes less than $100 \%$ efficient, the current wave begins to lag the voltage in direct proportion. The amount the current wave deviates from being $90^{\circ}$ out of phase with the voltage is defined as the dielectric loss angle. The tangent of this angle is known as the loss tangent or dissipation factor [19]. Hence a good dielectric film should have minimum dissipation factor. In the present study, the dissipation factor varies from 0.0011 to 0.004 and is independent of the deposition time. It almost remains constant for all the films with different deposition time.

\section{Conclusion}

AlN films have been prepared by DC reactive magnetron sputtering on Si (100) substrates for different deposition time. The evolution of preferred orientation and morphology of AIN films deposited at 50\% nitrogen concentration on Si (100) substrate was studied. The XRD analysis of the films revealed that, at a deposition time of $8 \mathrm{~min}$, the coated film favored the formation of highly oriented (002) preferential plane with enhanced crystal quality, which can provide good piezoelectric response. The optical reflectance spectra show reflectance at 240 $\mathrm{nm}$. The band gap increased with the increase in the deposition time and the values of refractive index were in the range of $1.1-1.2$, for the samples prepared at $8 \mathrm{~min}$ of deposition time. The MIS structures fabricated using AlN at different deposition time showed a significant improvement of electrical characteristics with deposition time as we go from $2 \mathrm{~min}$ to $8 \mathrm{~min}$.

\section{REFERENCES}

[1] B. Mednikarov, G. Spasov and T. Babeva, "Aluminum Nitride Layer Prepared by DC/RF Magnetron Sputtering," Journal of Optoelectronics and Advanced Materials, Vol. 7, No. 3, 2005, pp. 1421-1427

[2] T. P. Drusedau and J. Blasing, "Optical and Structural Properties of Highly $c$-Axis Oriented Aluminum Nitride Prepared by Sputter-Deposition in Pure Nitrogen," Thin Solid Films, Vol. 377-378, 2000, pp. 27-31. doi:10.1016/S0040-6090(00)01380-8

[3] X. H. Ji, S. P. Lau, G. Q. Yu, W. H. Zhong and B. K. Tay, "Structural Properties and Nanoindentation of AlN Films by a Filtered Cathodic Vacuum Arc at Low Temperature," Journal of Physics D: Applied Physics, Vol. 37, No. 10, 2004, p. 1472. doi:10.1088/0022-3727/37/10/009

[4] M. Clement, L. Vergara, J. Sangardor, E. Iborra and A. Sanz-Herras, "SAW Characteristics of AlN Films Sputtered on Silicon Substrates," Ultrasonic, Vol. 42, No. 1-9, 2004, pp. 403-407. doi:10.1016/j.ultras.2004.01.034

[5] T. Palacios, F. Calle, E. Monroy, J. Grajal, M. Eickhoff, O. Ambacher and C. Prieto, "Nanotechnology for SAW
Devices on AlN Epilayers," Materials Science and Engineering: $B$, Vol. 93, No. 1-3, 2000, pp. 154-158. doi:10.1016/S0921-5107(02)00022-3

[6] H. Gong and X. Jiang, "c-Axis Oriented AlN Films Prepared on Diamond Film Substrate by Electron Cyclotron Resonance Plasma Enhanced Chemical Vapour Depositon," Journal of Crystal Growth, Vol. 235, No. 1-4, 2000, pp. 333-339.

[7] A. J. Shuskus, T. M. Reeder and E. L. Paradis, "RF-Sputtered Aluminum Nitride Films on Sapphire," Applied Physics Letters, Vol. 24, No. 4, 1974, pp. 155-156. doi:10.1063/1.1655132

[8] M. Ishihara, K. Yamamoto, F. Kokai and Y. Koga, "Aluiminum Nitride Thin Films Prepared by Radical-Assisted Pulsed Laser Deposition," Vaccum, Vol. 59, No. 2-3, 2000, pp. 649-656. doi:10.1016/S0042-207X(00)00329-8

[9] L. Huang, X. D. Wang, K. W. Hipps, U. Mazur, R. Heffron and J. T. Dickinson, "Chemical Etching of Ion Beam Deposited AlN and AlN:H," Thin Solid Films, Vol. 279, No. 1-2, 1996, pp. 43-48.

[10] M. M. D. Ramos, J. B. Almeida, M. I. C. Ferrerira and M. P. D. Santos, "Thin Film Deposition by Magnetron Sputtering and Determination of Some Physical Parameters," Thin Solid Films, Vol. 176, No. 2, 1989, pp. 219-226. doi:10.1016/0040-6090(89)90095-3

[11] J. Huang, L. Wang, Q. Shen, C. Lin and O. Milae, "Preparation of AlN Films by Nitridation of Al Coated Si Sunstrate," Thin Solid Films, Vol. 340, No. 1-2, 1999, pp. 137-139. doi:10.1016/S0040-6090(98)01373-X

[12] V. Dimitrova, D. Monova, T. Paskova, Tz. Uzunov, N. Lvanoc and D. Dechev, "Aluminum Nitride Thin Films Deposited by DC Reactive Magnetron Sputtering FN2," Vaccum, Vol. 51, No. 2, 1998, pp. 161-164.

[13] M. Ishihara, K. Yamamoto, F. Kokai and Y. Koga, "Effect of Laser Wavelength for Surface Morphology of Aluminum Nitride Thin Films by Nitrogen Radical-Assisted Pulsed Laser Deposition," Japanese Journal of Applied Physics, Vol. 40, No. 575, 2001, pp. 2413-2416. doi: 10.1143/JJAP.40.2413

[14] H. Cheng, Y. Sun, J. X. Zhang, S. Yuan and P. Hing, "AlN Films Deposited under Various Nitrogen Concentrations by RF Reactive Sputtering," Journal of Crystal Growth, Vol. 254, No. 1-2, 2003, pp. 46-54. doi:10.1016/S0022-0248(03)01176-X

[15] X. H. Xu, C. J. Zhang and Z. H. Jin, "Morphological Properties of AIN Piezoelectric Thin Films Deposited by DC Reactive Magnetron Sputtering," Thin Solid Films, Vol. 388, No. 1-2, 2001, pp. 62-67. doi:10.1016/S0040-6090(00)01914-3

[16] Z. Q. Yao, Y. Q. Li, J. X. Tang, W. J. Zhang and S. T. Lee, "Growth and Photoluminescence Studies of AIN Thin Films with Different Orientation Degrees," Diamond and Related Materials, Vol. 17, No. 7-10, 2008, pp. 1785-1790. doi:10.1016/j.diamond.2008.02.009

[17] T. Adam, J. Kolodzey, C. P. Swann, M. W. Tsao and J. F. Rabolt, Vol. 175-176, 2001, pp. 428-435.

[18] V. Dimitrova, D. Manova and E. Valcheva, "Optical and Dielectric Properties of DC Magnetron Sputtered AIN 
Thin Films Correlated with Deposition Conditions," Materials Science and Engineering: B, Vol. 68, No. 1, 1999, pp. 1-4. doi:10.1016/S0921-5107(99)00221-4

[19] K. Jagannadham, A. K. Sharma, Q. Wei, R. Kalayanraman and J. Narayan, "Structural Characteristics of AlN
Films Deposited by Pulsed Laser Deposition and Reactive Magnetron Sputtering: A Comparative Study," Journal of Vac- uum Science \& Technology A, Vol. 16, No.5, 1998, pp. 2804-2816. doi:10.1116/1.581425 